\title{
Anandamide exerts its antiproliferative actions on cholangiocarcinoma by activation of the GPR55 receptor
}

\author{
Li Huang ${ }^{1,2,3}$, Jonathan C Ramirez ${ }^{1,4}$, Gabriel A Frampton ${ }^{1}$, Lessie E Golden ${ }^{1,4}$, Matthew A Quinn ${ }^{1,2}$, Hae Yong Pae , \\ Darijana Horvat ${ }^{1}$, Li-jian Liang ${ }^{3}$ and Sharon DeMorrow ${ }^{1,2,4}$
}

Cholangiocarcinomas are devastating cancers of biliary origin with limited treatment options. It has previously been shown that the endocannabinoid anandamide exerts antiproliferative effects on cholangiocarcinoma independent of any known cannabinoid receptors, and by the stabilization of lipid rafts, thereby allowing the recruitment and activation of the Fas death receptor complex. Recently, GPR55 was identified as a putative cannabinoid receptor; therefore, the role of GPR55 in the antiproliferative effects of anandamide was evaluated. GPR55 is expressed in all cholangiocarcinoma cells and liver biopsy samples to a similar level as in non-malignant cholangiocytes. Treatment with either anandamide or the GPR55 agonist, O-1602, reduced cholangiocarcinoma cell proliferation in vitro and in vivo. Furthermore, knocking down the expression of GPR55 prevented the antiproliferative effects of anandamide. Coupled to these effects was an increase in JNK activity. The antiproliferative effects of anandamide could be blocked by pretreatment with a JNK inhibitor and the lipid raft disruptors $\beta$-methylcyclodextrin and fillipin III. Activation of GPR55 by anandamide or 0-1602 increased the amount of Fas in the lipid raft fractions, which could be blocked by pretreatment with the JNK inhibitor. These data represent the first evidence that GPR55 activation by anandamide can lead to the recruitment and activation of the Fas death receptor complex and that targeting GPR55 activation may be a viable option for the development of therapeutic strategies to treat cholangiocarcinoma.

Laboratory Investigation (2011) 91, 1007-1017; doi:10.1038/labinvest.2011.62; published online 4 April 2011

KEYWORDS: cannabinoids; lipid rafts; JNK; Fas; G $\alpha 12$ G protein

Cholangiocarcinoma originates from the neoplastic transformation of epithelial cells that line the intra- and extrahepatic bile ducts. ${ }^{1,2}$ Symptoms are usually only evident after blockage of the bile duct, and at this late stage, chemotherapy and radiotherapy are relatively ineffectual leaving surgical resection as the only option for treatment. ${ }^{1,2}$ Because of this, these biliary cancers have a poor prognosis and improved treatments are urgently needed.

The endocannabinoid system was traditionally thought to consist of the cannabinoid receptors, $\mathrm{Cb} 1$ and $\mathrm{Cb} 2$, as well as a putative involvement of the vanilloid receptor (VR1), their endogenous ligands (endocannabinoids) and the proteins for their synthesis and inactivation. ${ }^{3}$ These cannabinoid receptors are seven-transmembrane-domain proteins coupled to $\mathrm{G}_{\mathrm{i} / \mathrm{o}}$ type of $\mathrm{G}$ proteins. ${ }^{3}$ Anandamide (AEA) was the first endogenous ligand to be identified, ${ }^{4}$ which acts as a partial Cb1 agonist and weak $\mathrm{Cb} 2$ agonist. A number of endocannabinoids, in particular AEA, are capable of mediating a plethora of cell signaling effects via an interaction lipid rafts; cholesterol-rich microdomains of the cell membrane; $;, 6$ as well as via the synthesis of the raftassociated, sphingolipid moiety, ceramide. ${ }^{7}$

More recently, GPR55 was also identified as a putative cannabinoid receptor $^{8,9}$ that is classified as a G $\alpha 12$ G-proteincoupled receptor. ${ }^{8}$ AEA has also been shown to function via GPR55, particularly with respect to controlling certain vascular endothelial cell functions. ${ }^{10}$ Furthermore, activation of $\mathrm{G} \alpha 12 \mathrm{G}$ proteins often leads to the activation of JNKmediated pathways. ${ }^{11}$

We have previously shown that AEA exerts growthsuppressing effects on cholangiocarcinoma by inducing apoptosis. ${ }^{12}$ This effect could not be blocked by Cb1, Cb2 or

\footnotetext{
${ }^{1}$ Department of Internal Medicine, Scott \& White Hospital and Texas A\&M Health Science Center College of Medicine, Temple, TX, USA; ${ }^{2}$ Digestive Disease Research Center, Temple, TX, USA; ${ }^{3}$ Department of Hepatobiliary Surgery, First Affiliated Hospital, Sun Yat-sen University, Guangzhou, China and ${ }^{4}$ Division of Research and Education Scott \& White Hospital, Temple, TX, USA

Correspondence: Dr S DeMorrow, PhD, Department of Internal Medicine, Scott \& White Hospital and Texas A\&M Health Science Center College of Medicine, Medical Research Building 702 SW HK Dodgen Loop, Temple, TX 76504, USA.

E-mail: demorrow@medicine.tamhsc.edu

Received 29 November 2010; revised 1 January 2011; accepted 31 January 2011
} 
VR1 antagonists, or by the specific $\mathrm{G}_{\mathrm{i} / \mathrm{o}}$ inhibitor, pertussis toxin. ${ }^{12}$ At the time, we assumed that AEA was acting via a receptor-independent mechanism. ${ }^{12}$ However, given the recent discovery and characterization of GPR55 as a novel AEA receptor, our data need to be reassessed to determine if GPR55 activation can decrease cholangiocarcinoma cell proliferation. In addition, we have previously shown that AEA-induced cell death is dependent on the stabilization of lipid rafts and the subsequent recruitment of the death receptor, Fas, into these structures. ${ }^{12}$ Thus, our aims are to determine if these AEA-mediated effects on cholangiocarcinoma cell growth can be attributed to the activation of GPR55.

\section{MATERIALS AND METHODS Cell Lines}

Four human cholangiocarcinoma cell lines Mz-ChA-1 (gift from Dr G Fitz, University of Texas Southwestern Medical Center, Dallas, TX, USA), and HuCC-T1, CCLP1 and SG231 (all from Dr AJ Demetris University of Pittsburgh, Pittsburgh, PA, USA) were used. ${ }^{13}$ The human immortalized, non-malignant cholangiocyte cell line (H69; from Dr GJ Gores, Mayo Clinic, Rochester, MN, USA) and the primary human intrahepatic cholangiocyte cell line (HIBEC; Sciencell, Carlsbad, CA, USA) were used as non-malignant control cell lines.

\section{Real-Time PCR}

RNA was extracted from all cell lines using the RNeasy Mini Kit (Qiagen Inc., Valencia, CA, USA) according to the instructions provided by the vendor and reverse transcribed using the Reaction Ready First Strand cDNA synthesis kit (SA Bioscience, Frederick, MD, USA). These reactions were used as templates for the PCR assays using a SYBR Green PCR master mix (SA Bioscience) in the real-time thermal cycler (ABI Prism 7900HT sequence detection system) using commercially available primers designed against human GPR55, G $\alpha 12$ and GAPDH (SA Bioscience). A $\Delta \Delta \mathrm{CT}$ analysis was performed using the untreated cells as the control sample. ${ }^{14,15}$ Data are expressed as relative mRNA levels \pm s.e.m. $(n=3)$.

\section{Immunoblotting}

Immunoblots to detect GPR55, G $\alpha 12$, flotillin-1, Fas and $\beta$-actin were performed as described previously, ${ }^{12}$ using specific antibodies against each protein. Where appropriate, data are expressed as fold change (mean \pm s.e.m.) of the relative expression after normalization with $\beta$-actin.

\section{Immunofluorescence}

Cells were seeded into six-well dishes containing a sterile coverslip on the bottom of each well. Cells were allowed to adhere overnight, washed once in cold PBS, fixed to the coverslip with $4 \%$ paraformaldehyde (in PBS) at room temperature for $5 \mathrm{~min}$, permeabilized in PBS containing
$0.2 \%$ Triton X-100 (PBST) and blocked in 4\% bovine serum albumin (in PBST) for $1 \mathrm{~h}$. GPR55 immunoreactivity was determined using a specific primary antibody (Genetex Inc., Irvine, CA, USA), followed by immunofluorescent detection using cy3-conjugated secondary antibodies (Jackson Immunochemicals, West Grove, PA, USA). Coverslips were mounted onto microscope slides with antifade gold containing 4',6-diamidino-2-phenylindole (DAPI) as a counterstain (Molecular Probes, Eugene, OR, USA). Negative controls were performed with the omission of the respective primary antibodies. Immunofluorescence was visualized using an Olympus IX-71 inverted confocal microscope.

\section{Immunohistochemistry}

Immunoreactivity for GPR55 was assessed in commercially available Accumax tissue arrays (Isu Abxis Co., Ltd, Seoul, Korea) by immunohistochemistry as described, ${ }^{16}$ using specific antibodies. These tissue arrays contain 46 cholangiocarcinoma biopsy samples from a variety of tumor differentiation grades as well as four control liver biopsy samples.

\section{MTS Cell Viability Assays}

Cell viability was evaluated by 3-(4,5-dimethylthiazol-2-yl)-5(3-carboxymethoxyphenyl)-2-(4-sulfophenyl)- $2 \mathrm{H}$-tetrazolium, inner salt (MTS) assay in the indicated cell lines. After trypsinization, cells were seeded into 96-well plates (10000 cells per well) in a final volume of $200 \mu \mathrm{l}$ of medium. Cells were stimulated with various concentrations of $\mathrm{O}-1602$ or AEA $\left(10^{-9}\right.$ to $\left.10^{-5} \mathrm{M}\right)$ for $48 \mathrm{~h}$ in the presence of specific inhibitors. The inhibitors utilized were the lipid raft disruptors, methyl- $\beta$-cyclodextrin $(0.1 \mathrm{mM}),{ }^{17}$ fillipin III $(1 \mathrm{mg} /$ $\mathrm{ml})^{18,19}$ and the JNK inhibitor $\left(10^{-7} \mathrm{M}\right.$; SP600125; EMD Chemicals, Gibbstown, NJ, USA).$^{20}$ Data were expressed as the fold change of treated cells compared with vehicle-treated controls. Statistical significance was determined using a $t$-test and $P<0.05$ was considered significant.

\section{Annexin V Staining}

Apoptosis was evaluated using Annexin-V labeling of cells. Cell lines were seeded in six-well plates (500000 cells per well) containing sterile coverslips on the bottom of each well and allowed to adhere overnight. Cells were stimulated with O-1602 or AEA (both at $10^{-5} \mathrm{M}$ ) for $24 \mathrm{~h}$, after which time coverslips were removed and rinsed in cold incubation buffer (10 mM HEPES, pH 7.4, $140 \mathrm{mM} \mathrm{NaCl}, 5 \mathrm{mM} \mathrm{CaCl}_{2}$ ) to remove excess media. Coverslips were then incubated in a solution containing Annexin V-biotin complex (1:50 dilution in incubation buffer) for $30 \mathrm{~min}$ at room temperature. After washing the coverslips to remove excess Annexin Vbiotin, the cells were fixed in cold 4\% PFA (in PBS) for $10 \mathrm{~min}$. Bound Annexin V-biotin complex was detected with Cy2-labeled streptavidin (1:200 dilution in PBS), mounted onto microscope slides with Prolong Antifade Gold containing DAPI and visualized using an Olympus IX-71 
inverted confocal microscope. The number of Annexin V-positive cells was counted and expressed as a percentage of total cells in eight random fields for each treatment group. Data are average \pm s.e.m. of eight fields in three independent experiments.

\section{Establishment of Stable Transfected Cell Lines}

Stable transfected cell lines were established using SureSilencing shRNA (SA Bioscience) plasmids for human GPR55 or G $\alpha 12$ G protein, which contain a marker for neomycin resistance for the selection of stably transfected cells following the methodology described previously. ${ }^{21}$ Subsequent clones were then assessed for the relative knockdown of the specific target genes using real-time PCR.

\section{Nude Mice Treatment}

In vivo experiments were performed in accordance with the guidelines of the Scott \& White IACUC committee. Mz-ChA-1 cells $\left(3 \times 10^{6}\right)$ were suspended in $0.25 \mathrm{ml}$ of extracellular matrix gel and injected subcutaneously in the flanks of these animals. After the establishment of the tumors, mice received O-1602 (10 mg/kg, i.p.) injected three times per week. In parallel, mice were injected with Mz-Neo-neg cells $\left(3 \times 10^{6}\right.$ cells) or Mz-GPR55 shRNA cells and treated with O-1602 $(10 \mathrm{mg} / \mathrm{kg}$, i.p.) or AEA $(10 \mathrm{mg} / \mathrm{kg}$, i.p.) three times per week.

Tumor tissues were excised from the flank of these mice at the conclusion of the study, fixed in formalin and embedded in paraffin. The cholangiocyte marker cytokeratin-19 (CK-19) was evaluated by immunohistochemical staining ${ }^{13}$ and GPR55 immunoreactivity was assessed by immunofluorescence. Apoptosis was detected in these sections using the ApopTag peroxidase in situ apoptosis detection kit following the manufacturer's instructions (Millipore, Temucula, CA, USA).

\section{JNK Activity Kit}

Phospho-JNK activity was assayed by ELISA (R\&D Systems, Minneapolis, MN, USA).

Mz-ChA-1, Mz-Neo-neg, Mz-GPR55 shRNA and $\mathrm{Mz}-\mathrm{G} \alpha 12$ shRNA cells were seeded in six-well plates at a concentration of 500000 cells per well. Cells were stimulated with AEA and O-1602 (both at $10^{-5} \mathrm{M}$ ) for various times up to $6 \mathrm{~h}$. Phospho-JNK activity levels for the cell lysates were assayed according to the manufacturer's specifications. All samples were assayed in triplicate, and phospho-JNK values obtained were normalized with total protein levels. Data are expressed as average \pm s.e.m., and statistical significance was determined using a $t$-test; $P<0.05$ was considered significant.

\section{Isolation of Lipid Raft Microdomains}

$\mathrm{Mz}$-ChA-1 cells were treated with AEA or O-1602 $\left(10^{-5} \mathrm{M}\right)$ in the absence or presence of the JNK inhibitor, SP600125 $\left(10^{-7} \mathrm{M}\right)$, for $24 \mathrm{~h}$. Cells were lysed and lipid rafts isolated by sucrose density centrifugation. ${ }^{12}$ In parallel, lipid rafts were fractionated from Mz-GPR55 shRNA and Mz-Neo-neg cells treated with AEA or O-1602. Fractions were collected and analyzed by western blotting as described previously using specific antibodies against flotillin-1, $\beta$-actin, GPR55, G $\alpha 12$ $\mathrm{G}$ protein and Fas.

\section{Colocalization Labeling with Lipid Raft Structures}

Lipid rafts were visualized using the Vybrant lipid raft labeling kit (Invitrogen, Carlsbad, CA, USA) after AEA or O-1602 $\left(10^{-5} \mathrm{M}\right)$ treatment for $24 \mathrm{~h}$ and were double labeled with Fas and GPR55 receptor antibodies as described. ${ }^{12}$ Coverslips were mounted onto microscope slides with prolong antifade gold containing DAPI and visualized using an Olympus IX-71 inverted confocal microscope.

\section{Statistical Analysis}

All data are expressed as mean \pm s.e.m. Differences between groups were analyzed by the Student's unpaired $t$-test when two groups were analyzed and ANOVA when more than two groups were analyzed, followed by an appropriate post hoc test. For the nude mice experiments where two parameters were variable (treatment and time) and two-way ANOVA analysis was performed, followed by the appropriate post hoc test. In each case, a $P$-value of less than 0.05 was used to indicate statistical significance.

\section{RESULTS}

\section{GPR55 Expression in Cholangiocarcinoma}

All of the cholangiocarcinoma cell lines studied here as well as the non-malignant cell lines (H69 and HIBEC) expressed the mRNA (Figure 1a) and protein (Figure 1b) for GPR55. There was no obvious or consistent difference between the GPR55 mRNA and protein expression in the malignant and non-malignant cell lines (Figure $1 \mathrm{a}$ and $\mathrm{b}$ ). By immunofluorescence, GPR55 immunoreactivity was predominantly found in the membrane and cytoplasm in all cell lines studied (Supplementary Figure S1A). Furthermore, immunohistochemical analysis of human liver biopsy samples indicated similar intensity and subcellular location of GPR55 immunoreactivity in malignant and non-malignant cholangiocytes (Supplementary Figure S1B).

\section{Activation of GPR55 Exerts Growth-Suppressive Effects on Cholangiocarcinoma In Vitro and In Vivo}

Activation of GPR55 with the specific agonist O-1602 in vitro significantly decreased cell viability in all cholangiocarcinoma cell lines studied, but not in the non-malignant cholangiocyte cell lines (Figure 2a). Furthermore, there was an increase in apoptosis in all cholangiocarcinoma cell lines after O-1602 treatment as shown by Annexin V staining (Supplementary Figure S2). Treating in vivo xenograft cholangiocarcinoma tumors with O-1602 significantly inhibited tumor growth (Figure 2b). In addition, the latency of tumor growth (ie, time taken for tumor volume to increase to $150 \%$ of the original size) was increased from $5.8 \pm 0.76$ to $11.2 \pm 2.74$ days after chronic O-1602 treatment. Histological analysis of 


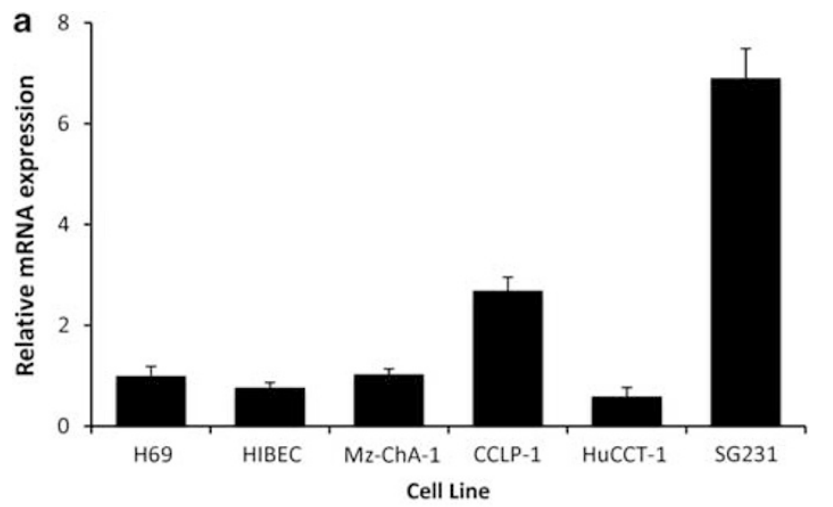

b

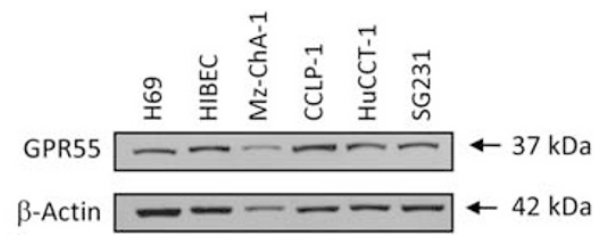

Figure 1 G-protein-coupled receptor 55 (GPR55) is expressed in cholangiocyte and cholangiocarcinoma cell lines. GPR55 levels were assessed in four cholangiocarcinoma cell lines as well as the non-malignant cholangiocyte cell lines $\mathrm{H} 69$ and HIBEC, by real-time polymerase chain reaction (PCR) (a) and immunoblotting (b). Data are expressed as average relative GPR55 mRNA expression ( \pm s.e.m.) in each cell line compared with H69 cells after using glyceraldehyde-3-phosphate dehydrogenase (GAPDH) expression as a loading control $(n=3$; a). the excised tumors revealed that all cancer cells within tumors from O-1602- and vehicle-treated animals were CK-19 positive, indicating a cholangiocyte phenotype (Supplementary Figure S3). Furthermore, all cholangiocarcinoma cells in the tumor retained GPR55 immunoreactivity, which appeared to increase after O-1602 treatment (Supplementary Figure S3). Using TUNEL staining as a marker of apoptosis, $\mathrm{O}-1602$ treatment increased the incidence of apoptosis in the cholangiocarcinoma tumors (Supplementary Figure S3).

\section{AEA Exerts Its Antiproliferative Actions Through GPR55 Activation}

We have previously shown that AEA exerts antiproliferative effects on cholangiocarcinoma ${ }^{12}$ in a manner similar to that shown here for GPR55 activation. Therefore, we wanted to assess definitively if AEA is working through a GPR55dependent mechanism. To this end, we stably knocked down the expression of GPR55 by transfecting GPR55-specific shRNA constructs into Mz-ChA-1 cells. Characterization of the resulting cell line (Mz-GPR55 shRNA) revealed a 60-70\% knock down in GPR55 mRNA and protein expression when compared with the mock-transfected cell line (Mz-Neo neg; Supplementary Figure S4). Using these cell lines, we assessed the antiproliferative effects of both AEA and O-1602 in vitro and in vivo. Both AEA and O-1602 decreased cell viability in $\mathrm{Mz}-\mathrm{Neo}$ neg to a similar degree, but failed to have any effect

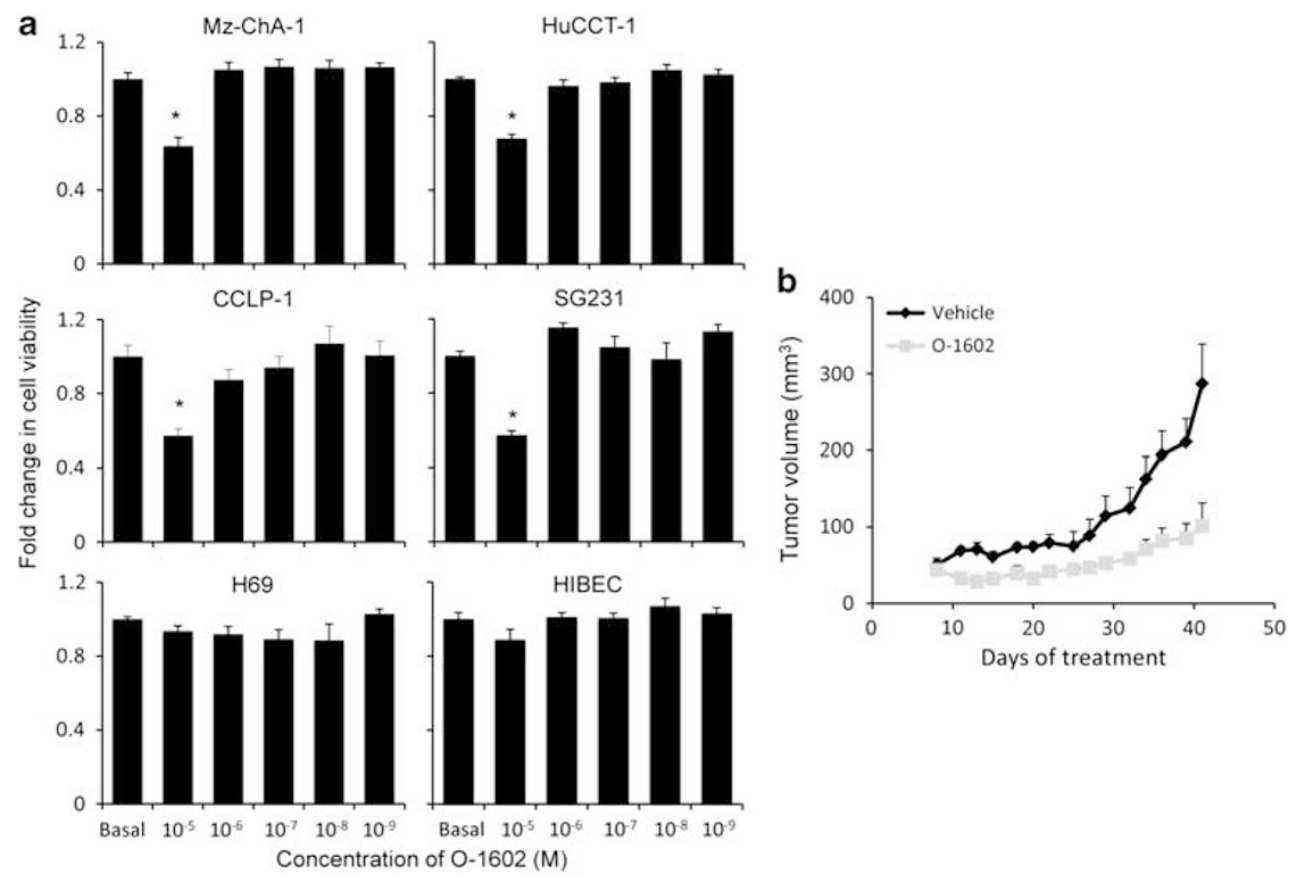

Figure 2 (a) Cholangiocarcinoma cells (Mz-ChA-1, HuCCT-1, CCLP-1 and SG231) and non-malignant H69 and HIBEC cells were treated with various concentrations of the G-protein-coupled receptor 55 (GPR55) agonist, O-1602 $\left(10^{-9}-10^{-5} \mathrm{M}\right)$, for $48 \mathrm{~h}$. Cell viability was assessed by 3-(4,5-dimethylthiazol-2yl)-5-(3-carboxymethoxyphenyl)-2-(4-sulfophenyl)-2H-tetrazolium, inner salt (MTS) assays. Data are expressed as fold change in viability (average \pm s.e.m., $n=7 ;{ }^{\star} P<0.05$ compared with basal treatment within each cell line). (b) Mz-ChA-1 cells were injected into the flank of athymic mice. After tumors were established, mice were treated with $10 \mathrm{mg}$ per $\mathrm{kg}$ per day (intraperitoneally (i.p.)) O-1602, 3 days per week, and tumor volume was assessed. 

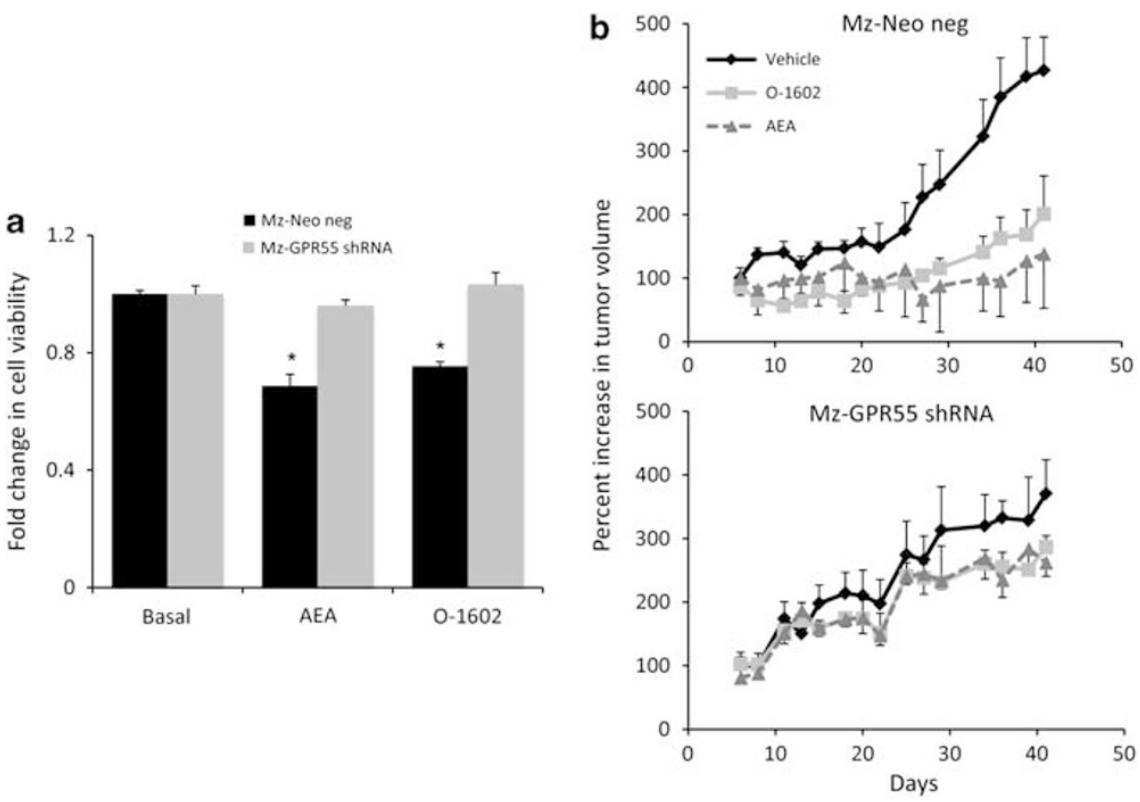

Figure 3 Specific knockdown of G-protein-coupled receptor 55 (GPR55) receptor expression prevents the antiproliferative effects of anandamide (AEA) in vitro and in vivo. Mz-ChA-1 cells were stably transfected with GPR55 short hairpin (sh)RNA vectors. The effect of AEA and O-1602 on cells with low levels of GPR55 expression (Mz-GPR55 short hairpin (sh)RNA) was assessed in vitro by 3-(4,5-dimethylthiazol-2-yl)-5-(3-carboxymethoxyphenyl)-2-(4sulfophenyl)-2H-tetrazolium, inner salt (MTS) assay (a). Mz-Neo-neg and Mz-GPR55 shRNA cells were treated with various concentrations of the GPR55 agonists, AEA and 0-1602 (10 $\left.10^{-5} \mathrm{M}\right)$, for $48 \mathrm{~h}$. Cell viability was assessed by MTS assays. Data are expressed as fold change in viability (average \pm s.e.m., $n=7$; ${ }^{*} P<0.05$ compared with basal treatment within each cell line). The effect of AEA and O-1602 on cells with low levels of GPR55 expression was assessed in vivo using the xenograft model of cholangiocarcinoma (b). Mz-Neo-neg cells and Mz-GPR55 shRNA cells were injected into the flank of athymic mice. After tumors were established, mice were treated with $10 \mathrm{mg}$ per $\mathrm{kg}$ per day (intraperitoneally (i.p.)) O-1602, 3 days per week, and tumor volume was assessed.

in Mz-GPR55 shRNA cells (Figure 3a). Similarly, AEA and O1602 increased the amount of apoptotic cells in Mz-Neo-neg cells, but had no effect in the Mz-GPR55 shRNA cells (Supplementary Figure S5). The growth-suppressive effects of AEA and O-1602 were evident in the xenograft model of cholangiocarcinoma using Mz-Neo-neg cells (Figure 3b), whereas tumors derived from the implantation of Mz-GPR55 shRNA were not sensitive to AEA or O-1602 (Figure 3b).

GPR55 is a G 12 G-protein-coupled receptor, ${ }^{8}$ and as such, we wanted to provide further evidence of the involvement of this receptor system in the antiproliferative actions of AEA. Again, we used a genetic approach and stably knocked down the expression of $\mathrm{G} \alpha 12$ by transfecting $\mathrm{G} \alpha 12$ specific shRNA constructs into Mz-ChA-1 cells. Characterization of the resulting cell line (designated $\mathrm{Mz}-\mathrm{G} \alpha 12$ shRNA) revealed a $60 \%$ knock down in both mRNA and protein expression compared with the Mz-Neo-neg cells (Supplementary Figure S6). We then assessed the effects of AEA and O-1602 on these cells in vitro and clearly showed that, as seen above, both AEA and O-1602 decreased cell viability in $\mathrm{Mz}-\mathrm{Neo}$ neg to a similar degree, but failed to have any effect in Mz-G $\alpha 12$ shRNA cells (Figure 4). Similarly, AEA and O-1602 increased the amount of apoptotic cells in Mz-Neo-neg cells, but had no effect in the Mz-G $\alpha 12$ shRNA cells (Supplementary Figure S7), suggesting a dependence on $\mathrm{G} \alpha 12$ for the antiproliferative effects of AEA and O-1602.

\section{GPR55 Activation Requires JNK Activity for Its Antiproliferative Effects}

One of the downstream consequences of the activation of G 12 G-protein-coupled receptors is the activation of JNK. ${ }^{11}$ We therefore assessed the effects of AEA and O-1602 on JNK activity. Treatment of Mz-ChA-1 cells with AEA and O-1602 increased JNK activity $1 \mathrm{~h}$ after stimulation, an effect that continued up to $6 \mathrm{~h}$ (Figure 5a). Furthermore, pretreatment of Mz-ChA-1 cells with the JNK inhibitor attenuated the antiproliferative effects of both AEA and O-1602 (Figure 5b). To show the requirement of GPR55 and G $\alpha 12$ in the AEAinduced activation of JNK activity, we assessed the effects of AEA and O-1602 on JNK activity in our knockdown cell lines. Treatment of the Mz-Neo-neg cells with AEA and O-1602 caused an increase in JNK activity in a manner similar to that seen in the parental cell line (Figure 5c). However, treatment of cells with reduced expression of either GPR55 (Mz-GPR55 shRNA) or G $\alpha 12$ (Mz-G $\alpha 12$ shRNA) with AEA or O-1602 failed to induce JNK activation (Figure $5 \mathrm{c}$ ). Taken together, these data suggest that the activation of GPR55 by AEA requires JNK activity to exert its antiproliferative effects in vitro.

\section{The Antiproliferative Effects of GPR55 Activation Requires Lipid Raft Structures}

We have previously shown that AEA exerts its tumorsuppressive effects via the stabilization of lipid raft structures 


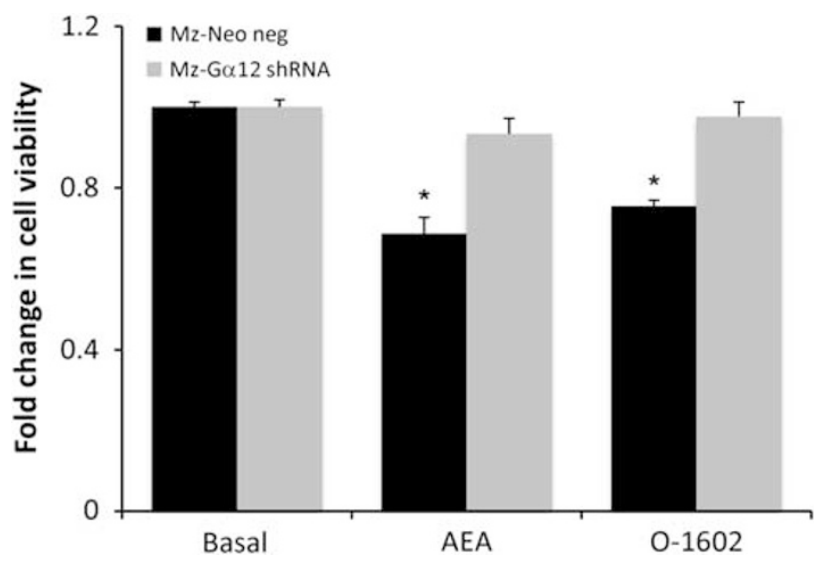

Figure 4 Specific knockdown of $\mathrm{G} \alpha 12 \mathrm{G}$-protein expression prevents the antiproliferative effects of G-protein-coupled receptor 55 (GPR55) activation in vitro. Mz-ChA-1 cells were stably transfected with $\mathrm{G} \alpha 12$ short hairpin (sh)RNA vectors ( $\mathrm{Mz}-\mathrm{G} \alpha 12$ shRNA). The effect of anandamide (AEA) and O-1602 on cells with low levels of $\mathrm{G} \alpha 12$ expression was assessed in vitro by 3-(4,5-dimethylthiazol-2-yl)-5-(3-carboxymethoxyphenyl)-2-(4-sulfophenyl)$2 \mathrm{H}$-tetrazolium, inner salt (MTS) assay. Mz-Neo-neg and Mz-G $\alpha 12$ shRNA cells were treated with various concentrations of the GPR55 agonist, O-1602 $\left(10^{-9}-10^{-5} \mathrm{M}\right)$, for $48 \mathrm{~h}$. Cell viability was assessed using an MTS cell proliferation assay. Data are expressed as fold change in viability (average \pm s.e.m., $n=7 ;{ }^{*} P<0.05$ compared with basal treatment within each cell line). and that disrupting lipid rafts prevents these antiproliferative effects. ${ }^{12}$ If our hypothesis that AEA is working via the activation of GPR55 is correct, the specific GPR55 agonist should also require lipid rafts for its function. To this end, cholangiocarcinoma cell lines were pretreated with the lipid raft disruptors, $\beta$-methyl cyclodextrin and fillipin III, before the addition of O-1602. Disruption of lipid rafts by these two agents prevented the antiproliferative effects of GPR55 activation in Mz-ChA-1 cells (Figure 6a) and the other cholangiocarcinoma cell lines studied (Supplementary Figure S8).

We then wanted to determine if GPR55 itself was recruited into lipid rafts or whether the lipid raft-mediated effects were downstream of receptor activation. Using sucrose density centrifugation, we isolated the detergent-resistant membrane fractions that contain the lipid raft structures. We have previously shown that under the conditions used in the experiments outlined here, lipid raft-enriched fractions can be found at the interface between the 5\% and 30\% sucrose layers, ${ }^{12}$ which corresponds to fractions 3 and 4 . Using flotillin-1 as a lipid raft marker, it can be seen that the lipid rafts did indeed float to the interface between 5 and 30\% sucrose layers under basal conditions and after AEA and O-1602 treatment, whereas the non-raft-associated $\beta$-actin

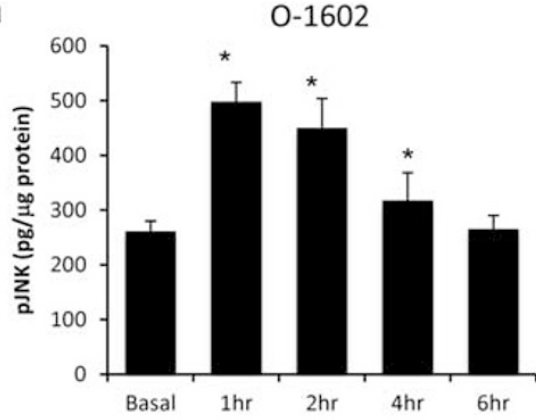

b

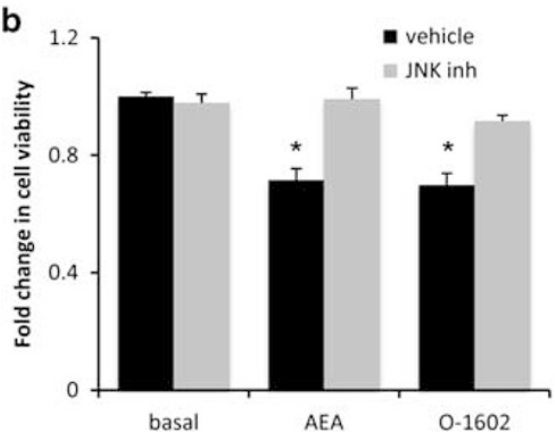

AEA
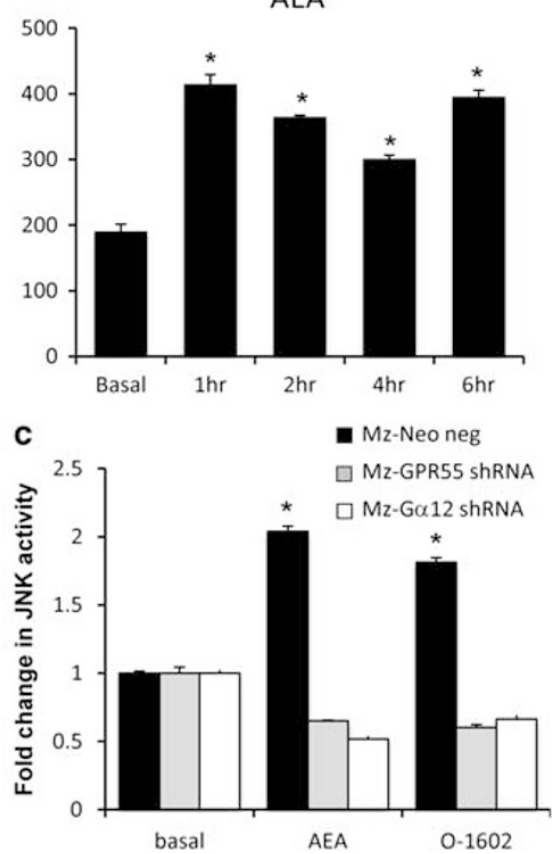

Figure 5 G-protein-coupled receptor 55 (GPR55) activation by anandamide (AEA) and O-1602 increases C-Jun N-terminal kinase (JNK) activity. (a) Mz-ChA-1

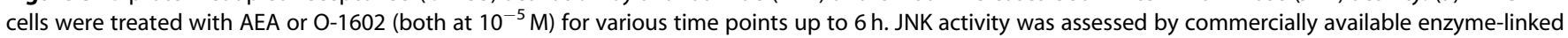
immunosorbent assay (ELISA) kits (average \pm s.e.m., $n=4 ;{ }^{\star} P<0.05$ compared with basal treatment). (b) Mz-ChA- 1 cells were pretreated with the JNK inhibitor, SP600125 (10 $\left.10^{-7} \mathrm{M}\right)$, for $1 \mathrm{~h}$ before the addition of AEA or 0-1602 (both at $\left.10^{-5} \mathrm{M}\right)$ for $48 \mathrm{~h}$. Cell viability was assessed by 3-(4,5-dimethylthiazol-2yl)-5-(3-carboxymethoxyphenyl)-2-(4-sulfophenyl)-2H-tetrazolium, inner salt (MTS) assays. Data are expressed as fold change in viability (average \pm s.e.m., $n=7 ;{ }^{\star} P<0.05$ compared with basal treatment within each cell line). (c) Mz-Neo-neg, Mz-GPR55 short hairpin (sh)RNA and Mz-G $\alpha 12$ shRNA cells were

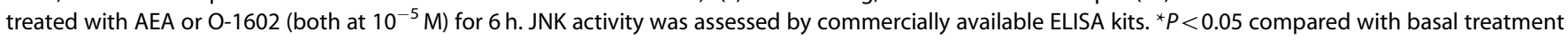
within each cell line. 
a

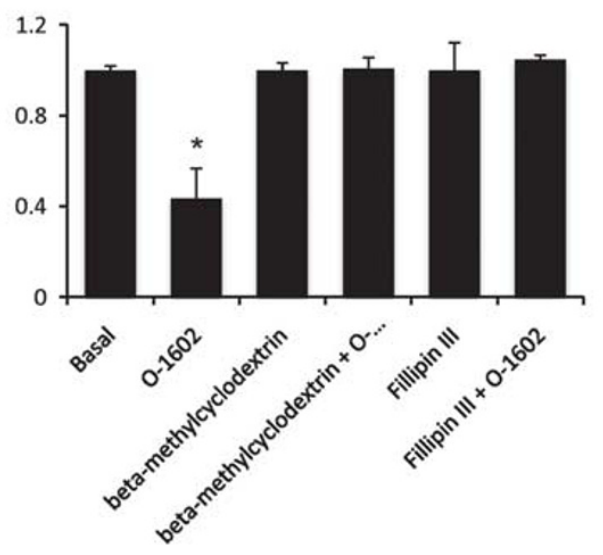

b

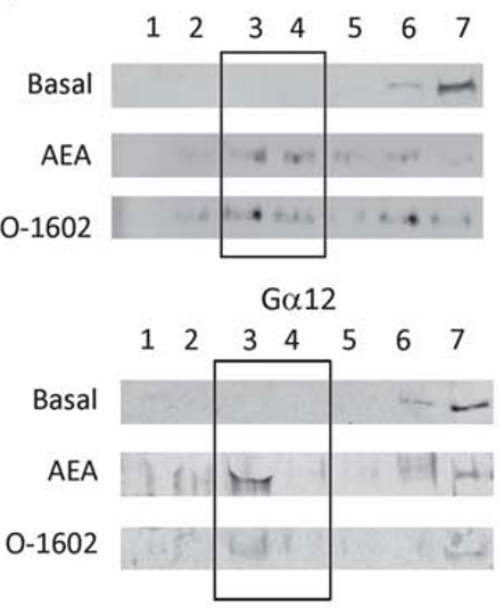

C

DAPI

GPR55

Lipid raft

merge

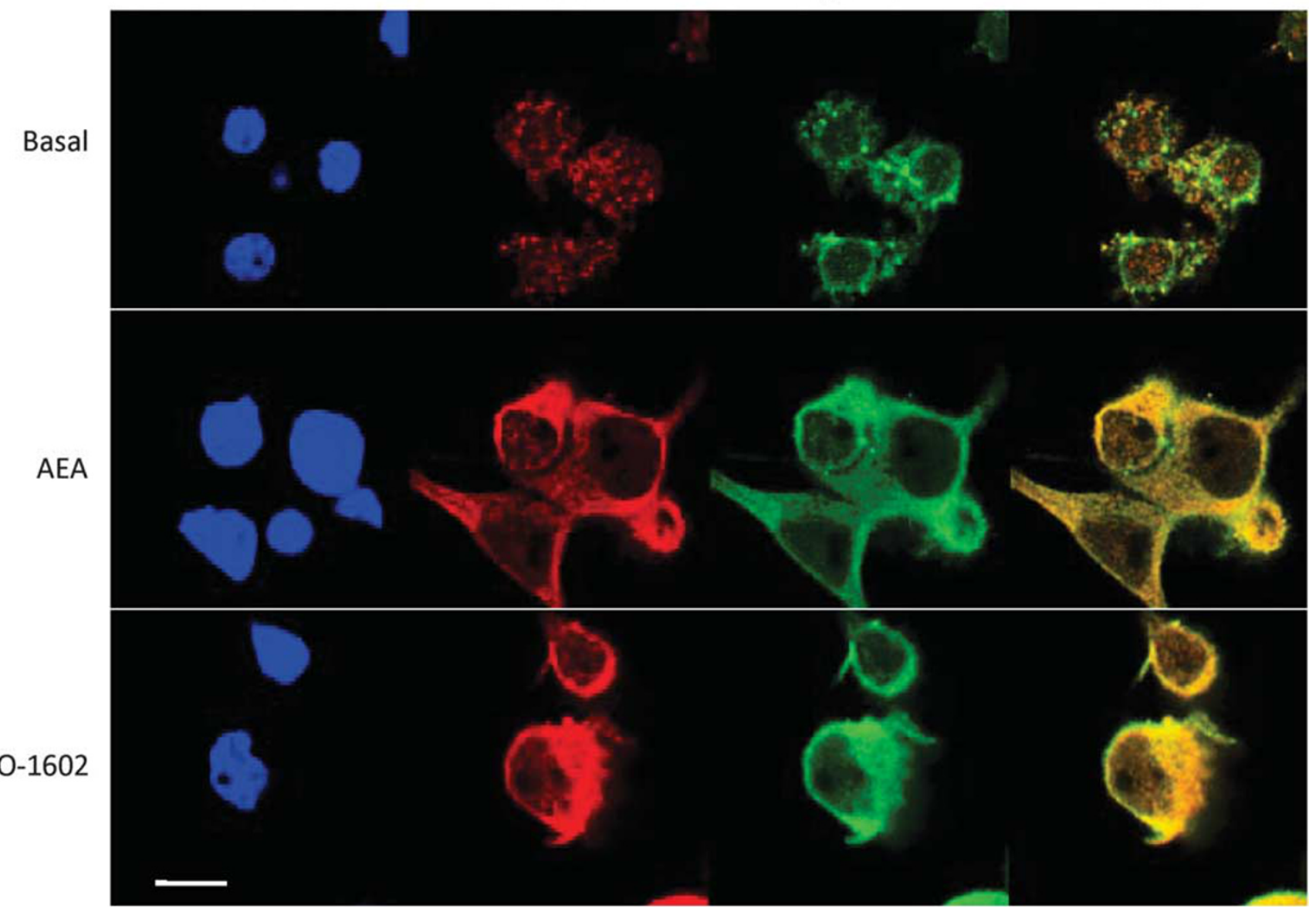

Figure 6 Disruption of lipid rafts inhibits the antiproliferative effects of G-protein-coupled receptor 55 (GPR55) activation. (a) Mz-ChA-1 cells were pretreated with lipid raft disrupters $0.1 \mathrm{mM} \beta$-methylcyclodextrin or $1 \mu \mathrm{g} / \mathrm{ml}$ fillipin III for $1 \mathrm{~h}$ before the addition of O-1602 (10 $\left.{ }^{-5} \mathrm{M}\right)$. Cell viability was determined by 3-(4,5-dimethylthiazol-2-yl)-5-(3-carboxymethoxyphenyl)-2-(4-sulfophenyl)-2H-tetrazolium, inner salt (MTS) assays. Data are expressed as average \pm s.e.m. $\left({ }^{*} P<0.05\right)$ compared with basal treatment $(n=7)$. (b) Mz-ChA-1 cells were treated with anandamide (AEA) or O-1602 (both at $\left.10^{-5} \mathrm{M}\right)$ for $4 \mathrm{~h}$ and detergent-resistant lipid rafts were isolated on a discontinuous $5-40 \%$ sucrose gradient. The resulting fractions were analyzed by immunoblotting using GPR55- and G $\alpha 12$-specific antibodies. (c) Colocalization of GPR55 immunoreactivity and lipid raft staining after AEA and O-1602 treatment. Mz-ChA-1 cells were treated with AEA and O-1602 (both at $10^{-5} \mathrm{M}$ ) and the lipid rafts were stained with Alexa Fluor 488-conjugated cholera toxin B subunit (green) as well as GPR55 immunoreactivity (red). Colocalization is indicated by yellow areas. Nuclei were counterstained with 4',6-diamidino-2-phenylindole (DAPI) (blue). Scale bar represents $10 \mu \mathrm{m}$. 
was used as a negative control to show that under our experimental conditions, non-raft-associated proteins, were not found in the lipid raft fractions (Supplementary Figure S9A). Using these characterized fractions, we showed that under basal conditions, GPR55 was exclusively found in the non-lipid raft fractions. However, after AEA and O-1602 treatment, GPR55 could be found in the lipid raft fractions as well (Figure 6b). This suggests that upon activation, GPR55 migrates into lipid rafts in the plasma membrane. As mentioned previously, GPR55 is coupled to G $\alpha 12$, and therefore if GPR55 migrates into lipid rafts, G $\alpha 12$ should also be found in lipid rafts. Indeed, $G \alpha 12$ was found predominantly in non-lipid raft fractions under basal conditions; however, after AEA and O-1602 treatment, G $\alpha 12$ was also found in lipid raft fractions (Figure 6b). Furthermore, $\mathrm{G} \alpha 12$ migration into lipid rafts after AEA and O-1602 treatment was observed in Mz-Neo-neg cells (Supplementary Figure S9B), but was absent in cells with GPR55 expression knocked down (Mz-GPR55 shRNA; Supplementary Figure S9B), suggesting a requirement for GPR55 expression for the translocation of $\mathrm{G} \alpha 12$ into lipid rafts in response to AEA or $\mathrm{O}-1602$ treatment.

In support of these data, double-staining experiments showed that under basal conditions there was a moderate overlap between GPR55 (red) and lipid raft-specific staining (green), indicated by the yellow color (Figure 6c). Pearson's correlation analysis of the degree of colocalization revealed a Pearson's coefficient in the range of $0.36-0.57$ in six random regions. However, after both AEA and O-1602 treatment, there was a greater degree of correlation and colocalization between GPR55 and lipid raft staining (Figure 6c), resulting in a Pearson's co-efficient in the range of 0.75-0.93.

\section{The Antiproliferative Effects of GPR55 Activation are the Result of Recruitment of Fas Death Receptor into Lipid Rafts}

We have previously shown that AEA appears to activate pro-apoptotic events in cholangiocarcinoma that require the involvement of the TNF super family, the recruitment of Fas and Fas ligand into lipid raft structures and the downstream effector molecule, Fas-associated death domain (FADD). ${ }^{12}$ Therefore, we wanted to assess if this phenomenon also occurred with the GPR55 agonists. Under basal conditions, Fas was found exclusively in the non-lipid raft fractions, but after AEA treatment, Fas could also be found in the lipid raft fractions (Figure 7a). Similarly, the recruitment of Fas into lipid raft fractions could also be detected after treatment with O-1602 (Figure 7a), suggesting that this recruitment of Fas into lipid rafts may be via GPR55 activation. a

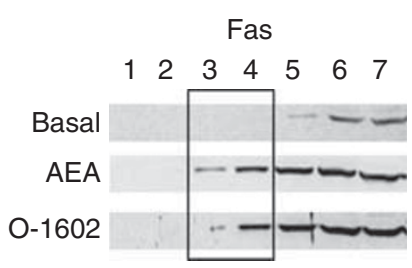

C

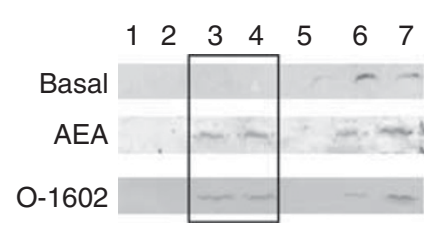

Mz-Neo neg

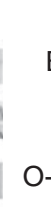

b

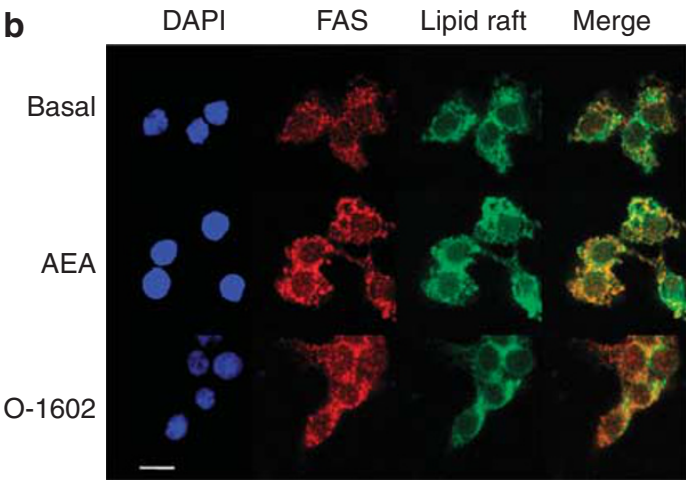

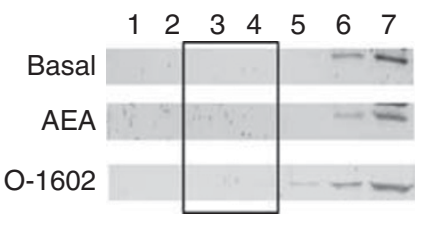

Mz-GPR55 shRNA

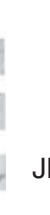

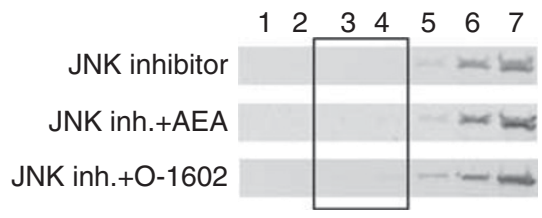

Figure 7 Fas receptor is recruited into lipid rafts after G-protein-coupled receptor 55 (GPR55) receptor activation. (a) Mz-ChA-1 cells were treated with anandamide (AEA) or O-1602 (both at $10^{-5} \mathrm{M}$ ) for $24 \mathrm{~h}$ and detergent-resistant lipid rafts were isolated on a discontinuous $5-40 \%$ sucrose gradient. The resulting fractions were analyzed by immunoblotting using a Fas-specific antibody to determine the subcellular location of these proteins. (b) Colocalization of Fas immunoreactivity and lipid raft staining after AEA and O-1602 treatment. Mz-ChA-1 cells were treated with AEA and O-1602 (both at $10^{-5} \mathrm{M}$ ) and the lipid rafts were stained with Alexa Fluor 488-conjugated cholera toxin B subunit (green) as well as Fas immunoreactivity (red). Colocalization is indicated by yellow areas. Nuclei were counterstained with 4',6-diamidino-2-phenylindole (DAPI) (blue). Scale bar represents $10 \mu \mathrm{m}$. (c) Mz-Neo-neg and Mz-GPR55 short hairpin (sh)RNA were treated with AEA or O-1602 (both at $10^{-5} \mathrm{M}$ ) for $24 \mathrm{~h}$ and detergent-resistant lipid rafts were isolated. In addition, Mz-ChA-1 cells were pretreated with the c-Jun N-terminal kinase (JNK) inhibitor, SP600125 $\left(10^{-7} \mathrm{M}\right.$ ), for $1 \mathrm{~h}$ before the addition of AEA or O-1602 (both at $10^{-5} \mathrm{M}$ ) for $24 \mathrm{~h}$, followed by lipid raft fractionation. The resulting fractions were analyzed by immunoblotting using a Fas-specific antibody to determine its subcellular location. 
In support of these data, colocalization experiments showed that under basal conditions there was a moderate overlap between Fas (red) and lipid raft-specific staining (green), indicated by the yellow color (Figure 7b). Pearson's correlation analysis of the degree of colocalization revealed a Pearson's coefficient in the range of $0.33-0.65$ in six random regions. However, after both AEA and O-1602 treatment, there was a greater degree of correlation and colocalization between Fas and lipid raft staining (Figure 7b), resulting in a Pearson's co-efficient of 0.74-0.94.

To clearly show the requirement of GPR55 expression in the AEA- and O-1602-mediated recruitment of Fas into lipid rafts, we evaluated this phenomenon in cells with suppressed GPR55 expression. As with the parental cell line, AEA and O-1602 treatment of mock-transfected cells (Mz-Neo neg) resulted in the recruitment of Fas into lipid raft structures (Figure 7c), whereas Fas recruitment into lipid rafts was not observed in Mz-GPR55 shRNA after AEA or O-1602 treatment (Figure 7c).

In addition, because we have shown that GPR55-mediated effects on cholangiocarcinoma cell viability require the activation of the JNK pathway, we wished to assess if the AEA- and O-1602-mediated recruitment of Fas into lipid rafts is also dependent on JNK activation. Indeed, pretreatment of cholangiocarcinoma cells with a JNK inhibitor prevented the translocation of Fas into lipid raft fractions after GPR55 activation (Figure 7c).

\section{DISCUSSION}

The major findings of this study relate to the endocannabinoid system as a potential therapeutic target aimed at regulating cholangiocarcinoma cell growth. We have previously shown that the antiproliferative and proapoptotic actions of AEA require lipid raft structures and depend on the translocation of Fas and FasL into these lipid raft structures. ${ }^{12}$ With the identification of GPR55 as a novel cannabinoid receptor capable of regulating the effects of AEA, ${ }^{8,9}$ we reassessed the mechanism by which AEA exerts its effects and have shown that: (1) both malignant and non-malignant cholangiocytes express GPR55 to a similar degree; (2) a specific GPR55 agonist has a similar suppressive effect on cholangiocarcinoma growth both in vitro and in vivo as AEA; (3) knocking down the expression of GPR55 prevents the antiproliferative action of AEA; and (4) the growthsuppressing effects of GPR55 activation by AEA requires G $\alpha 12$ and JNK activation and subsequent translocation of Fas into the lipid raft structures. These data suggest that GPR55 offers an intriguing target for the design of potential chemotherapeutic agents.

Consistent with our observation that AEA has antiproliferative and proapoptotic properties, cannabinoids of various origins (endogenous, plant-derived or synthetic analogues) have been shown to suppress cancer cell growth in vitro ${ }^{22-25}$ as well as in vivo. ${ }^{26}$ In addition to the lipid-raftmediated effects of AEA, we have shown that AEA induces a concomitant activation of the non-canonical Wnt pathway via upregulation of Wnt $5 \mathrm{a}^{15}$ as well as an increase in the proteolytic processing, and hence activation of the Notch 1 signaling pathway. ${ }^{27}$ How these seemingly independent observations fit together is a topic of ongoing research in our laboratory. The dependence and recruitment of the $\gamma$-secretase complex to lipid raft structures has previously been shown to modulate $\gamma$-secretase activity; ${ }^{28}$ therefore, it is conceivable that agents that stabilize or disrupt lipid raft structures such as cannabinoids ${ }^{12}$ may indeed also regulate the Notch signaling pathway. Furthermore, activation of the Wnt signaling pathway has been shown to overlap and crosstalk with the Notch signaling pathway. ${ }^{29-31}$ Indeed, activation of Notch 1 has been shown to upregulate the expression of Wnt5a in a number of cell models. ${ }^{30}$ The involvement of lipid rafts in the differential activation of the Notch signaling pathways by endocannabinoids and how the Wnt and Notch signaling pathways interact in these conditions are a topic of ongoing research in our laboratory.

In this study, we show that the antiproliferative actions of AEA, which we previously thought were cannabinoid receptor independent, ${ }^{12}$ are indeed through a GPR55dependent mechanism. The identification of GPR55 as a putative cannabinoid receptor is still contentious. Pharmacological evidence suggests that the interaction of AEA with GPR55 is cell-type and tissue-specific. ${ }^{32,33}$ Indeed, the majority of evidence identifying GPR55 as an AEA receptor was performed in endothelial cells. ${ }^{34}$ Little is known about the physiological role of GPR55 in the liver and is a topic of research in our laboratory.

It is interesting to note that the effects of AEA and O-1602 were not evident in the non-malignant cholangiocyte cell lines, even though GPR55 expression was found in malignant and non-malignant cells and tissues to a similar degree. There is a growing body of evidence indicating that cancer cells are enriched in cholesterol ${ }^{35-37}$ and display higher levels of cholesterol-rich lipid rafts than their non-malignant counterparts. $^{38}$ It is conceivable that because we have shown dependence on lipid rafts in the anti-tumoral effects of GPR55 receptor activation, the relative abundance of lipid raft structures between malignant and non-malignant cholangiocytes may be the reason behind the difference in susceptibility of these two cell types. Indeed, therapies that specifically induce lipid raft clustering and recruitment of apoptotic cascades (coined CASMERs; Clustering of Apoptotic Signaling Molecule-Enriched Rafts) are being developed as potential therapies for various cancers as they tend to be more effective in inducing apoptosis in cancer cells than in non-cancerous cells. ${ }^{39,40}$

The identification of GPR55 as a potential therapeutic target for the treatment of cancer is gaining momentum. Recently, GPR55 has been shown to regulate the growthpromoting effects of lysophosphatidylinositol in a number of tumor types ${ }^{41-43}$ via an increase in extracellular signal-regulated kinase cascade. ${ }^{41,42}$ Furthermore, GPR55 
expression in tumors from different origins correlates with their aggressiveness ${ }^{42}$ and its activation increases migration and invasive properties of breast cancer cells. ${ }^{43}$ In contrast, the data presented here suggest that upon activation with AEA, GPR55 has an antiproliferative and proapoptotic effect on cholangiocarcinoma cell growth. Presumably, these contradictory data are due to the ligand and subsequent downstream molecular events associated with GPR55 activation in this particular cell type and are a topic of further investigation in our laboratory.

Data, such as that presented here, showing the recruitment of protein components of the death receptor complexes into lipid raft structures are voluminous. It is widely accepted that both the TNF receptor complex and the Fas receptor complex are recruited into lipid rafts in response to apoptosisinducing drugs, which facilitates the receptor complex formation and is critical for their function. ${ }^{44}$ Indeed, we have previously shown that this recruitment into lipid rafts by cannabinoid stimulation occurs in cholangiocarcinoma cells via a mechanism that we previously thought to be cannabinoid receptor independent. ${ }^{12}$ In addition, these receptor complexes all require FADD to function. ${ }^{45}$ By overexpressing dominant-negative FADD, we blocked the antiproliferative effects of AEA, suggesting that the formation of these death receptor complexes is required for the AEA-mediated effects on cholangiocarcinoma cell growth. ${ }^{12}$ The data presented here confirm the recruitment of Fas receptor into lipid rafts by AEA, but clearly show a requirement for GPR55 expression in this event. Furthermore, our data also suggest that the recruitment of GPR55 and G $\alpha 12$ occurs upon receptor activation by AEA and O-1602. To our knowledge, this is the first demonstration of the recruitment of GPR55 into lipid rafts, but $G \alpha 12$ has previously been shown to be recruited via an interaction with heat-shock protein $90 .{ }^{46}$

In this study, we showed a role for JNK activation in the effects of AEA on cholangiocarcinoma cells, which is consistent with previous studies. ${ }^{47}$ In Chang liver cells, AEA administration activated JNK and resulted in the subsequent increase in AP1 DNA-binding activity and an increase in FasL expression. ${ }^{47}$ This effect was prevented by pretreatment with $\beta$-methyl cyclodextrin, ${ }^{47}$ which support the data presented here. How JNK activity leads to the recruitment of Fas into lipid rafts is unknown. One possibility may be that JNK, via its interaction with the chaperone heat-shock protein 90 , is recruited into lipid rafts, ${ }^{48}$ and somehow serves to stabilize lipid raft structures, thereby facilitating the death receptor complex formation. The lipid raft-stabilizing function of heat-shock proteins has previously been shown in neuronal cells. ${ }^{49}$ Another alternative may be that JNK signaling may merely increase the amount of Fas and FasL in the cells. ${ }^{47}$ Studies into the precise mechanism whereby JNK activation results in Fas recruitment into lipid raft structures are warranted.

In conclusion, we have clearly shown a role for GPR55 in the antiproliferative effects of AEA in vivo and in vitro.
Furthermore, GPR55 activation results in the recruitment of GPR55 and G $\alpha 12$ into lipid raft structures and subsequent activation of JNK. Downstream of these AEA-mediated effects was the recruitment of Fas into lipid rafts, which did not occur in cells with GPR55 or G $\alpha 12$ expression knocked down, nor after the pretreatment of cells with a JNK inhibitor. Taken together with our previous studies showing a requirement for Fas and FADD in the proapoptotic effects of AEA on cholangiocarcinoma cells, our data suggest that the stabilization of lipid raft structures by AEA facilitates the interaction between Fas and the other constituents of the Fas-containing death receptor complex, resulting in the induction of apoptosis in these cells. Cholangiocarcinoma has a very poor prognosis and survival rate; therefore, we propose that the development of novel therapeutic strategies that target GPR55 may prove beneficial for the treatment of this devastating disease.

Supplementary Information accompanies the paper on the Laboratory Investigation website (http://www.laboratoryinvestigation.org)

\section{ACKNOWLEDGEMENTS}

We thank Dr Andreea Trache and Ms Anna Webb of the Texas A\&M Health Science Center, Integrated Microscopy and Imaging Laboratory for assistance with confocal microscopy. We also thank Mr Akimuddin Mohamad and Mr Andrew Brennan for their technical assistance. This work was supported by an NIH K01 grant award (DK078532), an NIH R03 grant award (DK088012) and a Research Scholar award from the American Cancer Society (RSC 118760) to Dr DeMorrow, a state scholarship of China Scholarship Council to Dr Huang (2009638036), and a Scott \& White Mentored Research Award to Dr Ramirez.

\section{DISCLOSURE/CONFLICT OF INTEREST}

The authors declare no conflict of interest.

1. Blechacz BR, Gores GJ. Cholangiocarcinoma. Clin Liver Dis 2008;12: 131-150, ix.

2. Sirica AE. Cholangiocarcinoma: molecular targeting strategies for chemoprevention and therapy. Hepatology 2005;41:5-15.

3. Bisogno T, Ligresti A, Di Marzo V. The endocannabinoid signalling system: biochemical aspects. Pharmacol Biochem Behav 2005;81: 224-238.

4. Devane WA, Dysarz III FA, Johnson MR, et al. Determination and characterization of a cannabinoid receptor in rat brain. Mol Pharmacol 1988;34:605-613.

5. Hinz $\mathrm{B}$, Ramer $\mathrm{R}$, Eichele $\mathrm{K}$, et al. $R(+)$-methanandamide-induced cyclooxygenase-2 expression in $\mathrm{H} 4$ human neuroglioma cells: possible involvement of membrane lipid rafts. Biochem Biophys Res Commun 2004;324:621-626.

6. Siegmund SV, Uchinami $H$, Osawa $Y$, et al. Anandamide induces necrosis in primary hepatic stellate cells. Hepatology 2005;41: 1085-1095.

7. Mimeault M, Pommery N, Wattez $\mathrm{N}$, et al. Anti-proliferative and apoptotic effects of anandamide in human prostatic cancer cell lines: implication of epidermal growth factor receptor down-regulation and ceramide production. Prostate 2003;56:1-12.

8. Lauckner JE, Jensen JB, Chen HY, et al. GPR55 is a cannabinoid receptor that increases intracellular calcium and inhibits $M$ current. Proc Natl Acad Sci USA 2008;105:2699-2704.

9. Ryberg E, Larsson N, Sjogren $\mathrm{S}$, et al. The orphan receptor GPR55 is a novel cannabinoid receptor. Br J Pharmacol 2007;152:1092-1101.

10. Johns DG, Behm DJ, Walker DJ, et al. The novel endocannabinoid receptor GPR55 is activated by atypical cannabinoids but does not mediate their vasodilator effects. Br J Pharmacol 2007;152:825-831. 
11. Collins LR, Minden A, Karin M, et al. Galpha12 stimulates C-Jun NH2 terminal kinase through the small $G$ proteins Ras and Rac. J Biol Chem 1996;271:17349-17353.

12. DeMorrow $\mathrm{S}$, Glaser $\mathrm{S}$, Francis $\mathrm{H}$, et al. Opposing actions of endocannabinoids on cholangiocarcinoma growth: recruitment of fas and fas ligand to lipid rafts. J Biol Chem 2007;282:13098-13113.

13. Coufal M, Invernizzi $P$, Gaudio $E$, et al. Increased local dopamine secretion has growth-promoting effects in cholangiocarcinoma. Int J Cancer 2010;126:2112-2122.

14. Livak KJ, Schmittgen TD. Analysis of relative gene expression data using real-time quantitative PCR and the 2(-Delta Delta $C(T))$ method. Methods 2001;25:402-408.

15. DeMorrow S, Francis $H$, Gaudio $E$, et al. The endocannabinoid anandamide inhibits cholangiocarcinoma growth via activation of the noncanonical Wnt signaling pathway. Am J Physiol Gastrointest Liver Physiol 2008;295:G1150-G1158.

16. Alpini $G$, Invernizzi $P$, Gaudio $E$, et al. Serotonin metabolism is dysregulated in cholangiocarcinoma, which has implications for tumor growth. Cancer Res 2008;68:9184-9193.

17. Scheiffele $P$, Roth $M G$, Simons $K$. Interaction of influenza virus haemagglutinin with sphingolipid-cholesterol membrane domains via its transmembrane domain. EMBO J 1997;16:5501-5508.

18. Orlandi PA, Fishman PH. Filipin-dependent inhibition of cholera toxin: evidence for toxin internalization and activation through caveolae-like domains. J Cell Biol 1998;141:905-915.

19. Smart EJ, Ying YS, Conrad PA, et al. Caveolin moves from caveolae to the Golgi apparatus in response to cholesterol oxidation. J Cell Biol 1994;127:1185-1197.

20. Shin M, Yan C, Boyd D. An inhibitor of c-jun aminoterminal kinase (SP600125) represses c-Jun activation, DNA-binding and PMAinducible 92-kDa type IV collagenase expression. Biochim Biophys Acta 2002;1589:311-316.

21. Frampton GA, Lazcano EA, Li $\mathrm{H}$, et al. Resveratrol enhances the sensitivity of cholangiocarcinoma to chemotherapeutic agents. Lab Invest 2010;90:1325-1338.

22. De Petrocellis L, Melck D, Palmisano A, et al. The endogenous cannabinoid anandamide inhibits human breast cancer cell proliferation. Proc Natl Acad Sci USA 1998;95:8375-8380.

23. Ruiz L, Miguel A, Diaz-Laviada I. Delta9-tetrahydrocannabinol induces apoptosis in human prostate PC-3 cells via a receptor-independent mechanism. FEBS Lett 1999;458:400-404.

24. Sanchez $C$, de Ceballos ML, del Pulgar TG, et al. Inhibition of glioma growth in vivo by selective activation of the $\mathrm{CB}(2)$ cannabinoid receptor. Cancer Res 2001;61:5784-5789.

25. Sanchez C, Galve-Roperh I, Canova C, et al. Delta9-tetrahydrocannabinol induces apoptosis in C6 glioma cells. FEBS Lett 1998;436:6-10.

26. Galve-Roperh I, Sanchez C, Cortes ML, et al. Anti-tumoral action of cannabinoids: involvement of sustained ceramide accumulation and extracellular signal-regulated kinase activation. Nat Med 2000;6:313-319.

27. Frampton $\mathrm{G}$, Coufal M, Li H, et al. Opposing actions of endocannabinoids on cholangiocarcinoma growth is via the differential activation of Notch signaling. Exp Cell Res 2010;316:1465-1478.

28. Gamerdinger $M$, Clement $A B$, Behl $C$. Effects of sulindac sulfide on the membrane architecture and the activity of gamma-secretase. Neuropharmacology 2008;54:998-1005.

29. Collu GM, Brennan K. Cooperation between Wnt and Notch signalling in human breast cancer. Breast Cancer Res 2007;9:105.
30. Katoh M. Transcriptional mechanisms of WNT5A based on NF-kappaB, Hedgehog, TGFbeta, and Notch signaling cascades. Int J Mol Med 2009;23:763-769.

31. Nakamura T, Tsuchiya K, Watanabe M. Crosstalk between Wnt and Notch signaling in intestinal epithelial cell fate decision. J Gastroenterol 2007;42:705-710.

32. Sharir H, Abood ME. Pharmacological characterization of GPR55, a putative cannabinoid receptor. Pharmacol Ther 2010;126:301-313.

33. Brown AJ, Robin Hiley C. Is GPR55 an anandamide receptor? Vitam Horm 2009;81:111-137.

34. Waldeck-Weiermair M, Zoratti C, Osibow $\mathrm{K}$, et al. Integrin clustering enables anandamide-induced $\mathrm{Ca}^{2+}$ signaling in endothelial cells via GPR55 by protection against CB1-receptor-triggered repression. J Cell Sci 2008;121(Part 10):1704-1717.

35. Freeman MR, Solomon KR. Cholesterol and prostate cancer. J Cell Biochem 2004;91:54-69.

36. Tugnoli V, Tosi MR. Cholesteryl ester detection in a human urothelial carcinoma. Clin Chim Acta 2005;360:208-210.

37. Tosi MR, Tugnoli V. Cholesteryl esters in malignancy. Clin Chim Acta 2005;359:27-45.

38. Li YC, Park MJ, Ye SK, et al. Elevated levels of cholesterol-rich lipid rafts in cancer cells are correlated with apoptosis sensitivity induced by cholesterol-depleting agents. Am J Pathol 2006;168:1107-1118; quiz 1404-1105.

39. Mollinedo F, Gajate C. Lipid rafts and clusters of apoptotic signaling molecule-enriched rafts in cancer therapy. Future Oncol 2010;6: 811-821.

40. Mollinedo F, Gajate C. Lipid rafts, death receptors and CASMERs: new insights for cancer therapy. Future Oncol 2010;6:491-494.

41. Pineiro R, Maffucci T, Falasca M. The putative cannabinoid receptor GPR55 defines a novel autocrine loop in cancer cell proliferation. Oncogene 2011;30:142-152.

42. Andradas C, Caffarel MM, Perez-Gomez E, et al. The orphan G proteincoupled receptor GPR55 promotes cancer cell proliferation via ERK. Oncogene 2011;30:245-252.

43. Ford LA, Roelofs AJ, Anavi-Goffer S, et al. A role for L-alphalysophosphatidylinositol and GPR55 in the modulation of migration, orientation and polarization of human breast cancer cells. $\mathrm{Br} J$ Pharmacol 2010;160:762-771.

44. Muppidi JR, Tschopp J, Siegel RM. Life and death decisions: secondary complexes and lipid rafts in TNF receptor family signal transduction. Immunity 2004;21:461-465.

45. Ashkenazi A, Dixit VM. Death receptors: signaling and modulation. Science 1998;281:1305-1308.

46. Waheed AA, Jones TL. Hsp90 interactions and acylation target the $G$ protein Galpha 12 but not Galpha 13 to lipid rafts. J Biol Chem 2002;277:32409-32412.

47. Giuliano M, Calvaruso G, Pellerito $\mathrm{O}$, et al. Anandamide-induced apoptosis in Chang liver cells involves ceramide and JNK/AP-1 pathway. Int J Mol Med 2006;17:811-819.

48. Nieto-Miguel T, Gajate C, Gonzalez-Camacho F, et al. Proapoptotic role of Hsp90 by its interaction with c-Jun $\mathrm{N}$-terminal kinase in lipid rafts in edelfosine-mediated antileukemic therapy. Oncogene 2008;27:1779-1787.

49. Chen S, Bawa D, Besshoh S, et al. Association of heat shock proteins and neuronal membrane components with lipid rafts from the rat brain. J Neurosci Res 2005;81:522-529. 